\title{
Adaptive Nonlinear Flight Control of STOL-Aircraft Based on Incremental Nonlinear Dynamic Inversion
}

\author{
Y. Beyer* and A. Kuzolap ${ }^{\dagger}$ and M. Steen \\ Institute of Flight Guidance, Technische Universität Braunschweig, 38108 Braunschweig \\ J. H. Diekmann ${ }^{\S}$ and N. Fezans $s^{\text {II }}$ \\ Institute of Flight Systems, German Aerospace Center, 38108 Braunschweig
}

\begin{abstract}
In this paper an adaptive control system for a passenger aircraft with active high-lift system is presented. Failures in the high-lift system parts of such aircraft are critical and consequently need to be handled automatically. An adaptive controller is proposed which consists of incremental nonlinear dynamic inversion (INDI) with a reference model and linear controller. As the INDI is adaptive against uncertainties or system failures, no additional adaptive element like a neural network is needed. The implementation of the INDI requires a nonlinear system model which is permanently linearized during the runtime in order to obtain the current input matrix which here basically consists of the control surfaces effectiveness. It also requires feedback of the translational and rotational acceleration measurements which usually suffer from noise. In order to test the adaptivity of the INDI, a partial failure of the high-lift system during the landing approach is regarded. It shows that the INDI is capable of compensating the error by only using the conventional control surfaces.
\end{abstract}

Formular symbols

\section{Nomenclature}

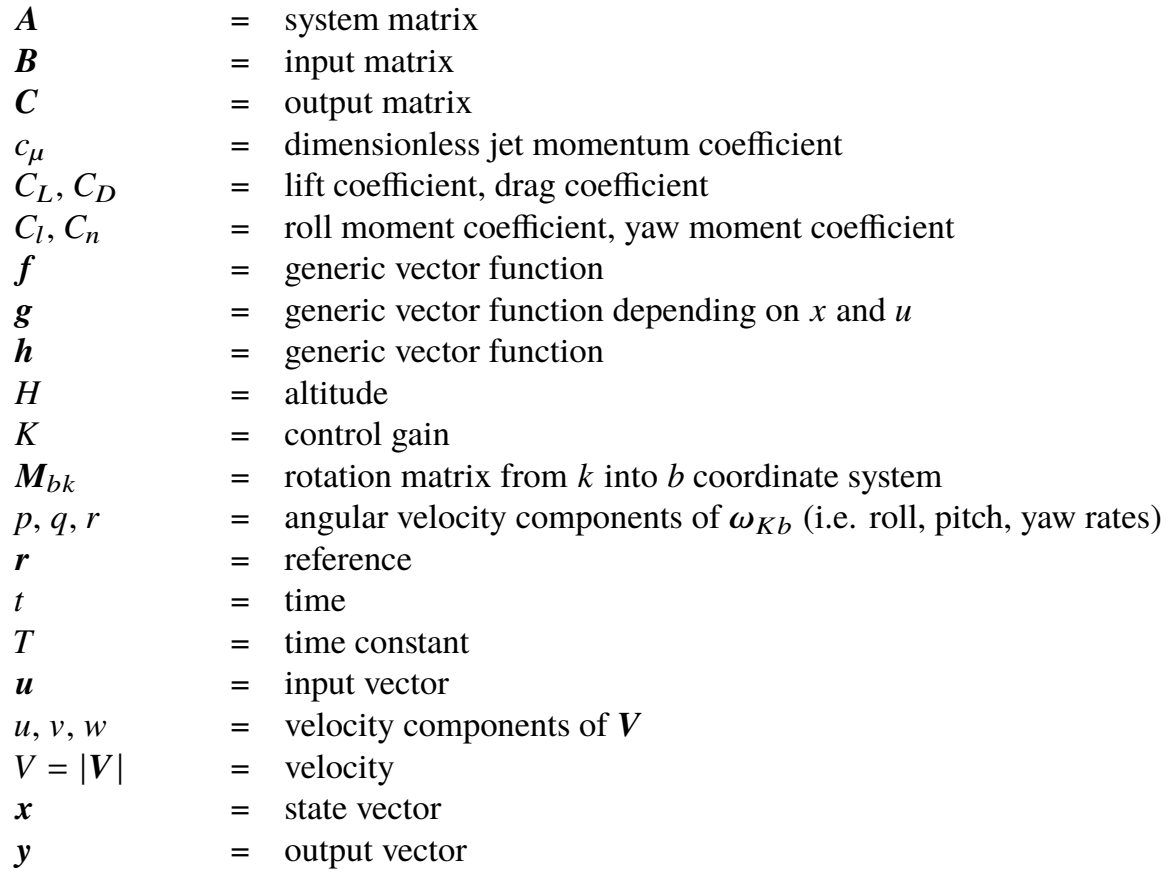

\footnotetext{
*Research Associate, Ph.D. Student, y.beyer@tu-bs.de.

${ }^{\dagger}$ Research Associate, Ph.D. Student, a.kuzolap@tu-bs.de.

$\doteqdot$ Senior Researcher, m.steen@tu-bs.de.

${ }^{\S}$ Research Scientist, jobst.diekmann@dlr.de.

IIScientific Advisor, nicolas.fezans@dlr.de
} 


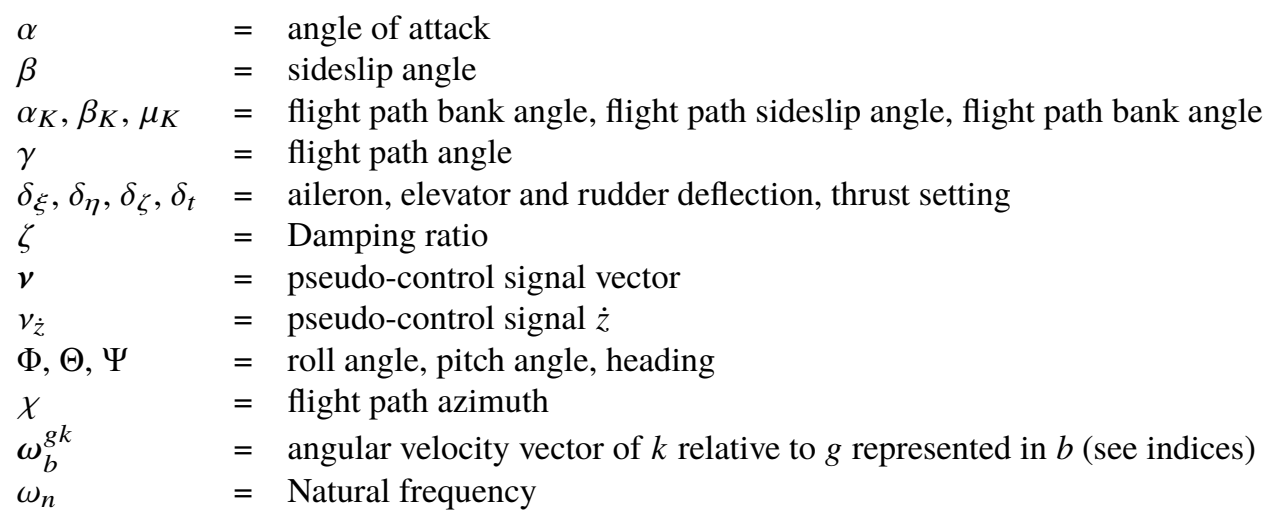

\section{Indices}

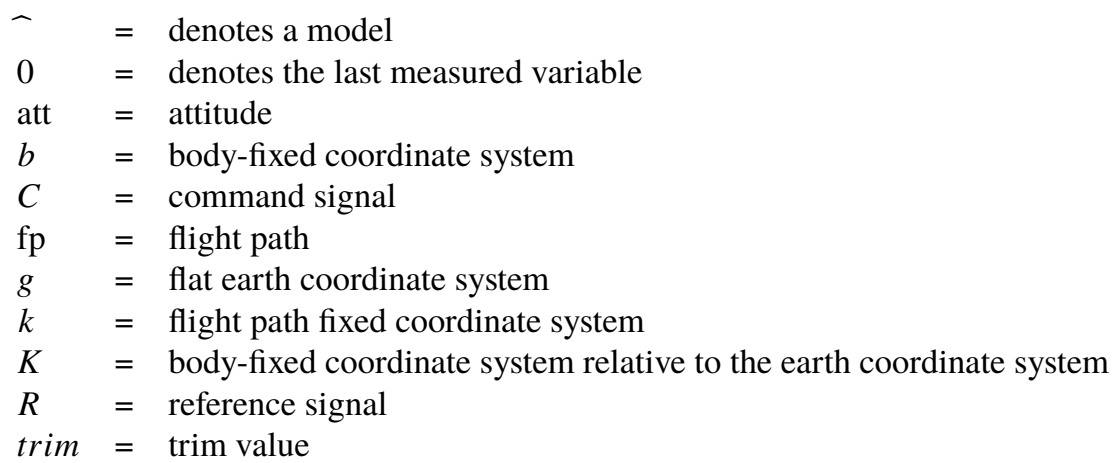

\section{Introduction}

Dassenger aircraft with active high-lift system are currently being researched within the Collaborative Research Penter SFB 880 for application at regional airports close to urban areas [1]. Such short takeoff and landing (STOL) aircraft are able to operate at very low airspeed. Consequently, the necessary starting and landing field length is reduced. Moreover, due to aerodynamic optimization as well as high climbing and descending ratios, noise pollution on the ground is reduced. Thus, the air transportation capacity could be increased [2]. An illustration of the SFB880 aircraft is shown in Fig. 1

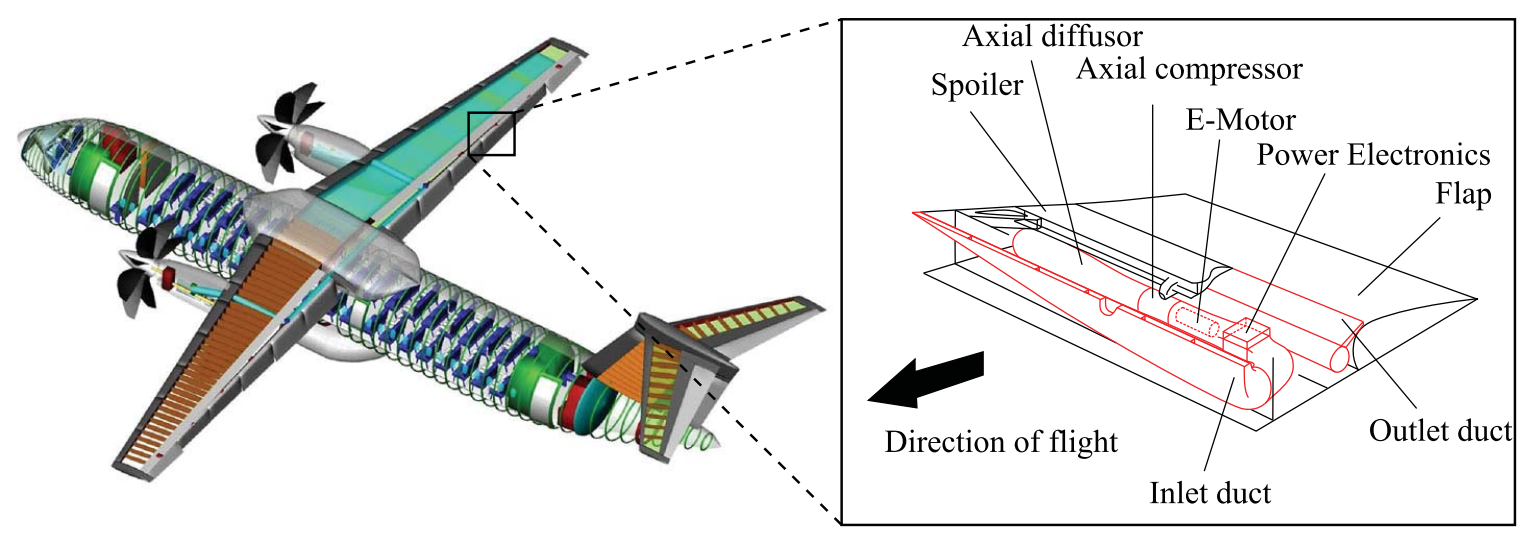

Fig. 1 Cutaway drawing of the SFB 880 reference aircraft and active high-lift system integration [3] 


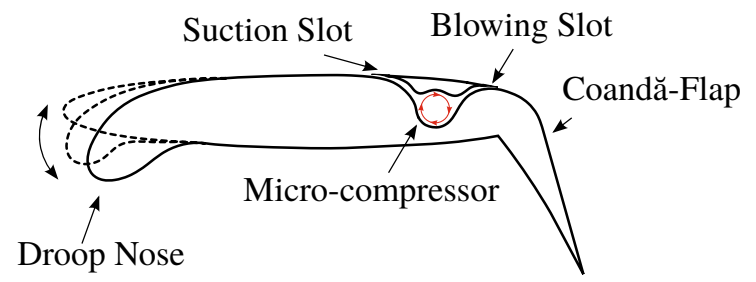

Fig. 2 Schematic diagram of the SFB 880 wing profile and the active high-lift system integration [4].

Due to multiple reasons, the flight physics of aircraft with active high-lift system is complex and highly nonlinear. This is mainly due to coupled aerodynamic propeller, wing and vertical tail interaction which results in an unstable lateral-directional motion during the approach [4]. As a consequence, a nonlinear controller stabilizing this instability is essential. The latter and other aerodynamic effects were previously researched [1, 5, 8$]$ and are briefly presented in section III]

Additional complexity arises from the realization of the active high-lift system illustrated in 2 The investigated active high-lift system is driven by twelve compressors arranged inside the wings along the wing. The tangential blowing combined with a single-hinged plain flap utilizes the Coandă effect [9]. The nonlinear and considerable impact of each compressor power setting on the aerodynamic coefficients is presented in section III.

During the approach flight phase, a failure of one of the high-lift compressors is critical. Due to the aforementioned nonlinearities, piloting the aircraft in case of a compressor failure is a demanding task requiring very active piloting (high workload as well as quick and precise reactions). To ensure stabilization of the aircraft, especially during the approach and critical situations, an adaptive nonlinear controller is proposed in order to handle the nonlinearities and to provide robustness against model uncertainties, operating point changes, and configuration changes, or system failures, is advantageous. In contrast to an adaptive controller, a non-adaptive robust scheme could result in a more conservative approach if the model uncertainties are too large [10].

A common strategy in the recent years for adaptive nonlinear aircraft control has been an implementation of a nonlinear dynamic inversion (NDI) in combination with an adaptive element that compensates the existing inversion error and system failures [10-14]. The inversion errors arise due to the lack of possibility to invert an exact model of the entire aircraft including the aerodynamics with a high model fidelity. Especially for the high-lift system, only an approximated analytical aerodynamic model is used that inherently causes inversion errors. To compensate those inversion errors, a neural network could be used [10-18]. An adaptive nonlinear controller is assumed to increase the safety of such aircraft which is investigated in this work.

However, the certification process of this type of control scheme in combination with neural networks is still a challenge. There exists some guidance for verification and validation and methods in high assurance systems with neural networks [19, 20], but this type of adaptive control systems is limited in terms of practical applicability. It is difficult to prove that the controller will rarely "learn incorrectly" under reasonable assumptions and if the adaptive element is able to recover when it has learned incorrectly to an extreme level [21]. Moreover, an NDI usually does not cover non-affine systems and its implementation effort increases the more complex the system is. To avoid these problems, an incremental NDI (INDI) is applied to control the STOL aircraft. This approach is adaptive to system uncertainties without neural network and suitable for non-affine systems. Previously, the INDI was used on fixed-wing aircraft flight control in [22,-25].

The controller theory and implementation is presented in section IV] the simulation results of a failure scenario with adaptive controller are presented and discussed in section $\mathrm{V}$

\section{Overview of the Flight Dynamic Model}

The detailed flight dynamic model of the STOL aircraft with active high-lift system is based on computational fluid dynamics (CFD) results and reference guide models. The model has a six degrees of freedom (6-DOF) rigid-body motion, three degrees of freedom in translation and rotation respectively. The calculation of the aerodynamic coefficients and variables was realized by a subdivision into the main components of the aircraft where each part adds a contribution to the total force and moment. The aerodynamic coefficients are calculated using the DLR TAU code [26] for specific operating conditions whose results are implemented as lookup tables or regression functions. Not only the conventional actuators but also the twelve compressors are available for the control of the aircraft. However, only the conventional controls are used in this study. The modeled dynamic actuator properties are given in Table 1 where $\omega_{n}$ denotes 


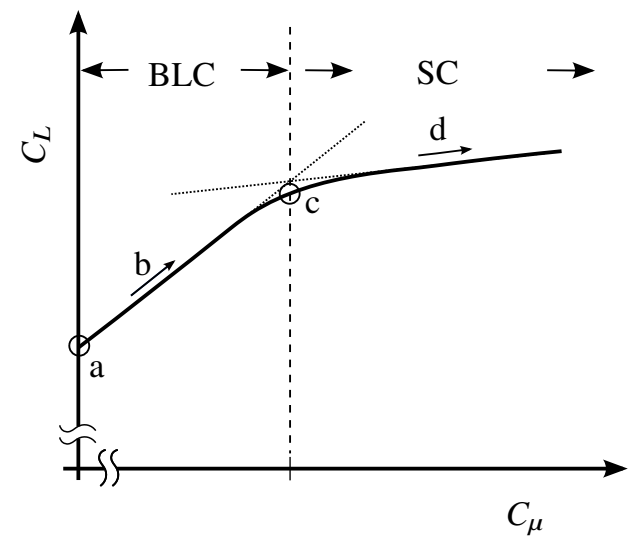

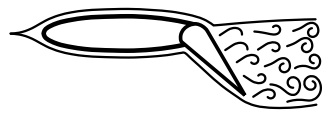

(a) Separated flow

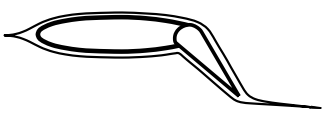

(c) Fully attached flow

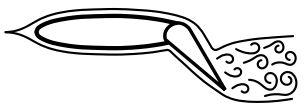

(b) Semi-attached flow

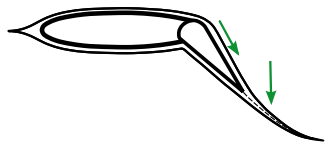

(d) Supercirculation

Fig. 3 Flow transition for blown flaps [4].

the natural frequency, $\zeta$ the daming ratio, $\delta_{\text {max }}$ the minimum and maximum deflection and $\dot{\delta}_{\text {max }}$ the minimum and maximum deflection rate. A detailed description of the model briefly presented here can be found in [4, 27. 29] where it is also extensively analyzed.

Table 1 Dynamic actuator properties

\begin{tabular}{cccccc}
\hline & time delay & $\omega_{n}$ & $\zeta$ & $\delta_{\max }$ & $\dot{\delta}_{\text {max }}$ \\
\hline aileron & $0.09 \mathrm{~s}$ & $60 \mathrm{~Hz}$ & 0.7 & $\pm 40^{\circ}$ & $\pm 40^{\circ} / \mathrm{s}$ \\
\hline elevator & $0.09 \mathrm{~s}$ & $60 \mathrm{~Hz}$ & 0.7 & $\pm 30^{\circ}$ & $\pm 40^{\circ} / \mathrm{s}$ \\
\hline rudder & $0.09 \mathrm{~s}$ & $60 \mathrm{~Hz}$ & 0.7 & $\pm 30^{\circ}$ & $\pm 40^{\circ} / \mathrm{s}$ \\
\hline
\end{tabular}

The active high-lift system utilizes a blowing impulse which is characterized by the dimensionless jet momentum coefficient $C_{\mu}$.

According to Fig. 3, the lift coefficient increases due to the jet momentum coefficient by the fact that the jet influences the boundary layer so that the flow separation point, on the upper side of the flap, moves towards the flap trailing edge with increasing $C_{\mu}$. This is the so-called "boundary layer control" (BLC) in the area of (b) in Fig. 3 . A further increase of the jet momentum coefficient causes a smaller increase of the lift and drag coefficient resulting from the increase of circulation which is in the so called area of supercirculation in the area of (d). The design jet momentum coefficient is supposed to be in the area of (c).

This aircraft exhibits some unconventional behavior which was already observed with various STOL aircraft in the past (e.g. [30]) but, to the best of the authors' knowledge, remained unexplained until the works of [4, 27]. The main cause for this behavior is the complex interaction between the propeller slipstream and the aft fuselage as well as the vertical stabilizer as illustrated by the streamlines in Fig. 4. It shows the flow around the configuration with a sideslip angle of $\beta=5.0^{\circ}$, an angle of attack of $\alpha=0.0^{\circ}$, a velocity of $V=54 \mathrm{~m} / \mathrm{s}$ and blowing with a dimensionless jet momentum coefficient of $C_{\mu}=0.033$ at steady flight condition thrust during approach. The interaction between the propeller slipstream and the aft fuselage as well as the vertical stabilizer causes unstable lateral-directional flight dynamics at low airspeeds. This effect is reduced for larger angles of attack and is related to the strong deflection of the flow through the flap deflection of $65^{\circ}$. 


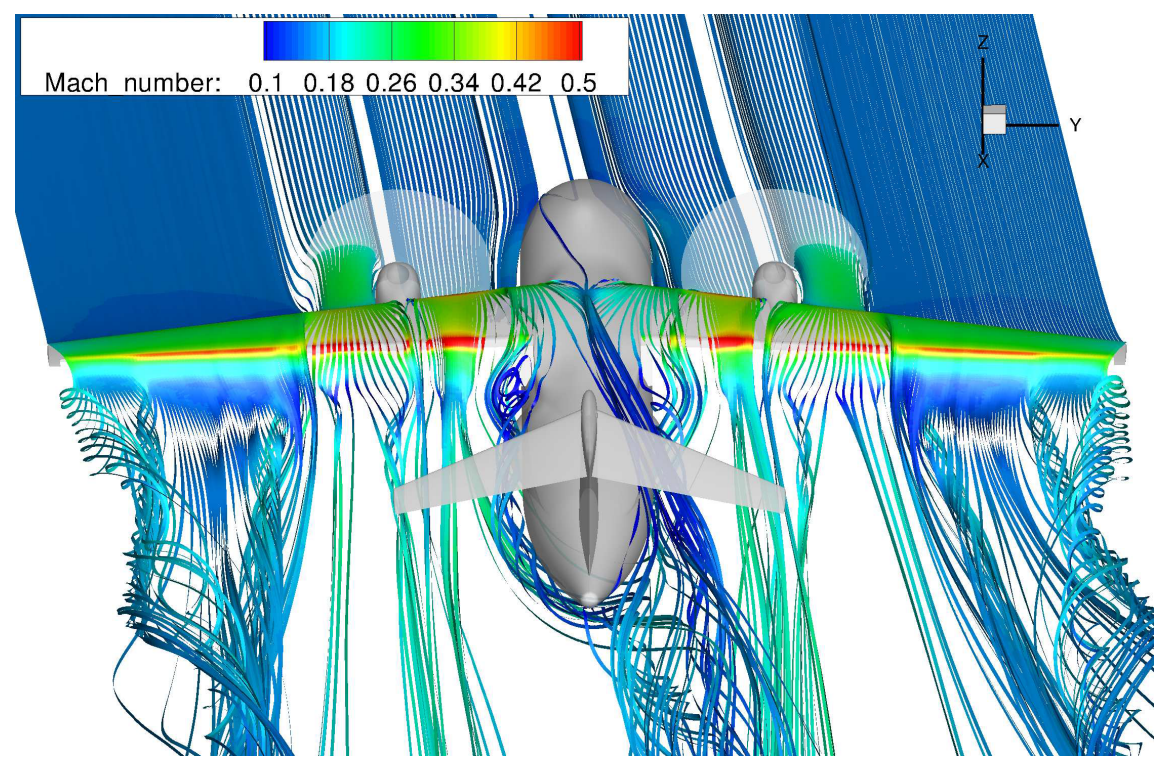

Fig. 4 Flow around the aircraft with sideslip angle $\beta=5.0^{\circ}$, angle of attack $\alpha=0.0^{\circ}$, velocity $v \approx 54 \mathrm{~m} / \mathrm{s}$ and a modarate thrust level [31].

The pole map of linear approximations of the nonlinear system depending on the airspeed is illustrated in Fig. 5 . Regarding the longitudinal motion, the short period mode (SP) is well damped. However, the natural frequency of the SP is relatively small. In combination with a relatively small dependency of the lift on the angle of attack, the reactions of the aircraft to a pitch command are rather sluggish but still acceptable. This could be compensated by direct lift control using the active high-lift system [27]. The phugoid $(\mathrm{Ph})$ is also stable and always acceptable. Regarding the lateral-directional motion, the poles of the Dutch roll (DR) as well as the spiral (S) are highly depending on the airspeed. For very low airspeeds, the flight dynamics are almost similar compared to those of conventional aircraft. However, the spiral diverges too fast to be acceptable. For slightly higher airspeeds, when the active high-lift system is still in use, the spiral becomes extremely unstable due to the interaction of the propeller and the aft of the fuselage, like shown in Fig. 4

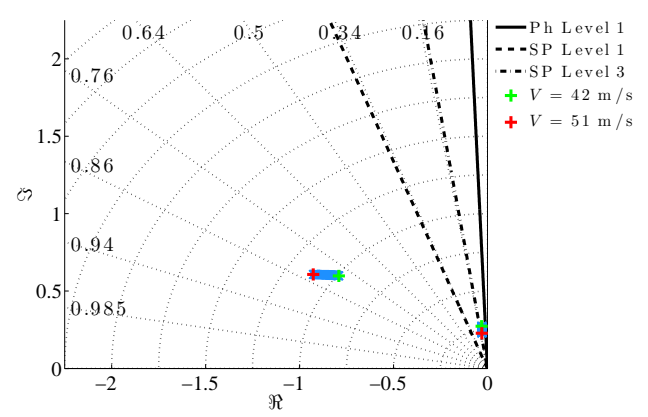

(a) Longitudinal motion

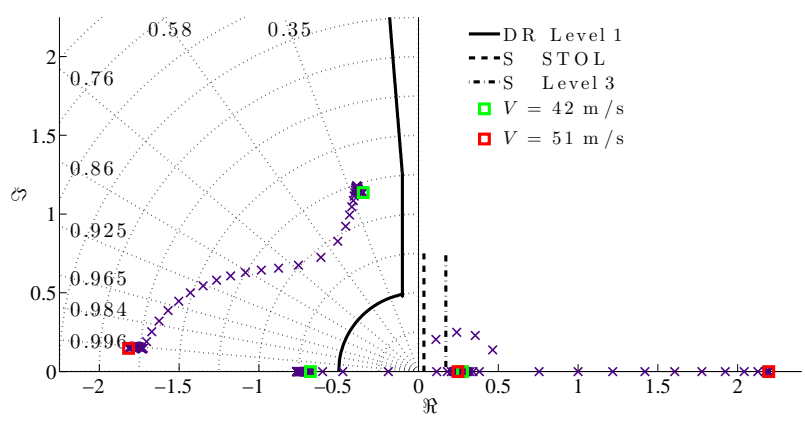

(b) Lateral-directionl motion

Fig. 5 Pole map for $V=42 \mathrm{~m} / \mathrm{s}$ until $51 \mathrm{~m} / \mathrm{s}$ at $H=300 \mathrm{~m}$ and $\beta=0^{\circ}$ [27].

For an accurate simulation of partial failures of the active high-lift system a model of the spanwise lift distribution, depending on each compressor power setting was developed [32]. The individual compressor jet momentum coefficient $\boldsymbol{C}_{\mu, \text { comp }}$ is used whereas the mean of the individual coefficient $\overline{\boldsymbol{C}}_{\mu, \text { comp }}=C_{\mu}$. This model is based on numerical aerodynamic simulations performed with the DLR TAU code [26]. For this investigation, this model structure is used based on latest aerodynamic data sets generated in the SFB 880. For instance, it now includes the influence of the droop 
nose and of the pylon. Moreover, the design jet momentum coefficient has slightly decreased from $C_{\mu}=0.0330$ to $C_{\mu}=0.0306$. As a result of these developments, the design lift coefficient is slightly higher and the relative drop of the lift coefficient due to a micro-compressor failure has increased. The applied model and data is illustrated in figure 6 where $C_{\mu}=0.0306$ is the design jet momentum coefficient. A failure of the fourth compressor shows a loss of local lift coefficient $C_{L}$ in the area of the compressor. Thus, also the roll moment coefficient $C_{l}$ decreases. Besides, the drag coefficient $C_{D}$ and the yaw moment coefficient $C_{n}$ decrease. The green line shows an example of six local jet momentum coefficients generated by individual settings of the six micro-compressors based on the spanwise model.
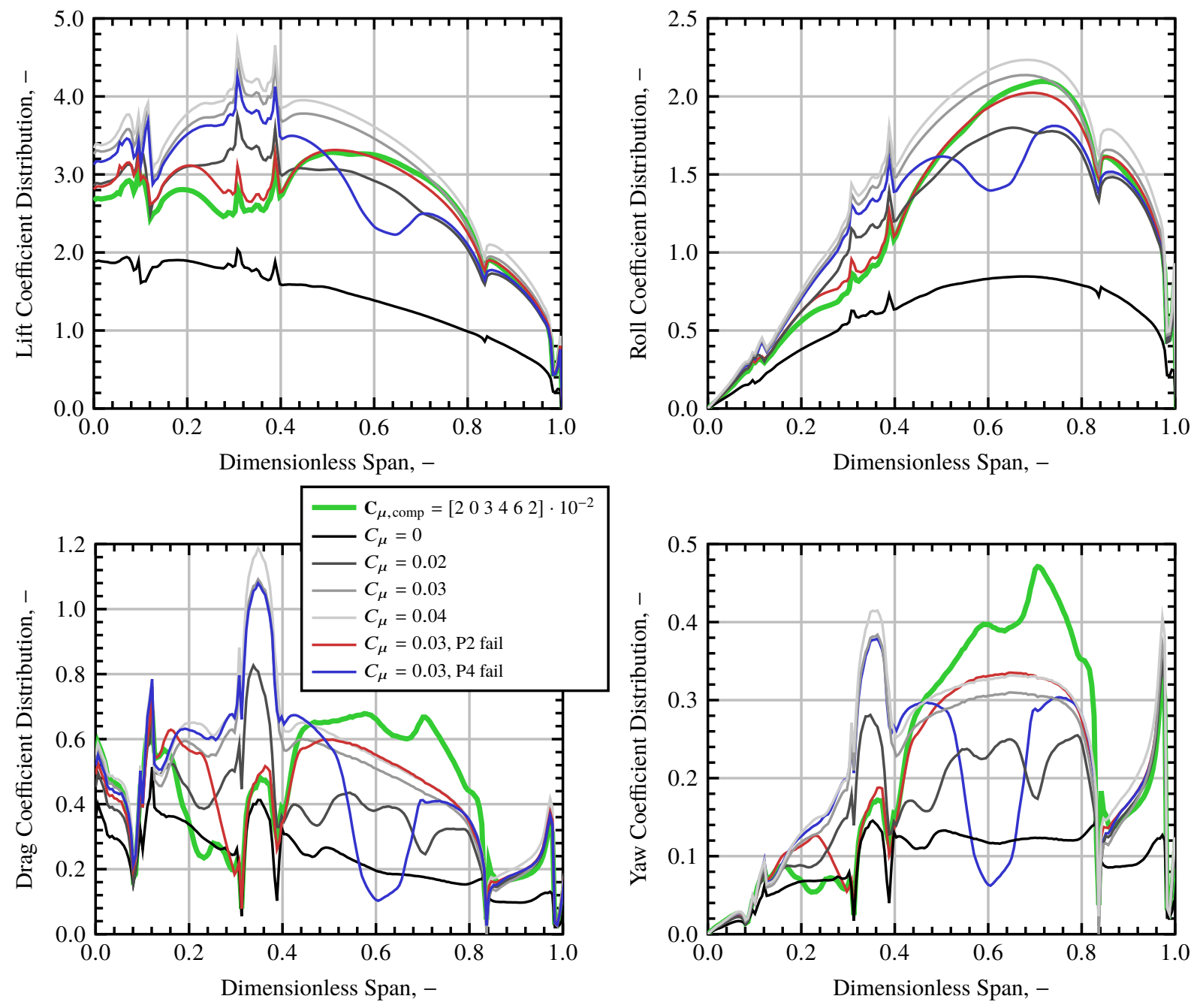

Fig. 6 Example of one spanwise aerodynamic coefficient distribution for arbitrary micro-compressor settings (green line) depending on six independently controllable micro-compressors based on numerical aerodynamic computations of specific operating points (other lines).

The lower the airspeed of the aircraft the more critical a partial failure of the active high-lift system becomes. At lower airspeed the control surface effectiveness is reduced, as it is proportional to the square of the airspeed. Additionally, the angle of attack is higher at lower airspeed (assuming that the value of the jet momentum coefficient has not been changed) and hence closer to the stall. 


\section{Basic Control Strategy}

\section{A. Incremental Nonlinear Dynamic Inversion}

The purpose of a feedback with Nonlinear Dynamic Inversion (NDI) is to linearize the input/output behavior of a nonlinear plant that allows the application of established linear control strategies [33, 34] without gain scheduling. The gain scheduling approach is to consider multiple linear plant approximations of different operating points and to design one linear controller for each of these points. However, due to high multidimensional nonlinearities, the number of the required points could be immense and the controller does not consider the nonlinearities in between or outside of these points. To show the main principle of NDI, a nonlinear multiple-input and multiple-output (MIMO) system is considered:

$$
\begin{aligned}
& \dot{x}=f(x)+g(x, u), \\
& y=h(x),
\end{aligned}
$$

where $\boldsymbol{f}$ and $\boldsymbol{g}$ are vector fields in the domain $D \subset \mathbb{R}^{n}$ and $\boldsymbol{x}, \boldsymbol{g}$ as well as $\boldsymbol{h}$ are LiPschitz, so that they are smooth in $D$. In order to realize the NDI, the output $\boldsymbol{y}$ or a differentation of $\boldsymbol{y}$ has to be obtained that it is directly controllable by the control input $\boldsymbol{u}$, where the number of derivatives describes the relative degree of the system. Each derivative is assigned to a new state vector and the last derivative is given by a nonlinear expression to complete the transformation. The last derivative is the new input or control signal of the inverted system which is called pseudo control signal $v$. There is the possibility to realize a system with a higher relative degree as a cascaded system, each with a relative degree of one [18, 22].

The idea of incremental NDI (INDI) is to consider linearized approximations of the nonlinear system during the runtime of the controller [35]. The relative degree of the nonlinear system in Eq. (1) is assumed to be one, which means that the control input $\boldsymbol{u}$ appears in the first derivative of $\boldsymbol{y}$. The linear approximation of Eq. (1) is

$$
\begin{aligned}
& \dot{\boldsymbol{x}} \approx \dot{\boldsymbol{x}}_{0}+\left.\frac{\partial}{\partial \boldsymbol{x}}(f(x)+g(x, u))\right|_{x=x_{0}, u=u_{0}}\left(x-x_{0}\right)+\left.\frac{\partial}{\partial \boldsymbol{u}}(g(x, u))\right|_{x=x_{0}, u=u_{0}}\left(u-u_{0}\right) \\
& y \approx y_{0}+\left.\frac{\partial}{\partial \boldsymbol{x}}(\boldsymbol{h}(\boldsymbol{x}))\right|_{x=x_{0}}\left(x-x_{0}\right),
\end{aligned}
$$

which is the linear state-space representation

$$
\begin{aligned}
& \dot{\boldsymbol{x}}-\dot{\boldsymbol{x}}_{0}=\boldsymbol{A}_{0}\left(\boldsymbol{x}-\boldsymbol{x}_{0}\right)+\boldsymbol{B}_{0}\left(\boldsymbol{u}-\boldsymbol{u}_{0}\right), \\
& \boldsymbol{y}-\boldsymbol{y}_{0}=C_{0}\left(\boldsymbol{x}-\boldsymbol{x}_{0}\right),
\end{aligned}
$$

where

$$
\begin{aligned}
& A_{0}=\left.\frac{\partial}{\partial \boldsymbol{x}}(f(\boldsymbol{x})+\boldsymbol{g}(\boldsymbol{x}, \boldsymbol{u}))\right|_{\boldsymbol{x}=\boldsymbol{x}_{0}, \boldsymbol{u}=\boldsymbol{u}_{0}}, \\
& \boldsymbol{B}_{0}=\left.\frac{\partial}{\partial \boldsymbol{u}}(\boldsymbol{g}(\boldsymbol{x}, \boldsymbol{u}))\right|_{\boldsymbol{x}=\boldsymbol{x}_{0}, \boldsymbol{u}=\boldsymbol{u}_{0}}, \\
& \boldsymbol{C}_{0}=\left.\frac{\partial}{\partial \boldsymbol{x}}(\boldsymbol{h}(\boldsymbol{x}))\right|_{\boldsymbol{x}=\boldsymbol{x}_{0}} .
\end{aligned}
$$

The state-space representation in Eq. (3) can be rewritten as

$$
\dot{\boldsymbol{y}}=\frac{\partial \boldsymbol{h}(\boldsymbol{x})}{\partial \boldsymbol{x}} \dot{\boldsymbol{x}} \approx \boldsymbol{C}_{0}\left(\dot{\boldsymbol{x}}_{0}+\boldsymbol{A}_{0}\left(\boldsymbol{x}-\boldsymbol{x}_{0}\right)+\boldsymbol{B}_{0}\left(\boldsymbol{u}-\boldsymbol{u}_{0}\right)\right)
$$

where $\boldsymbol{C}_{0} \dot{\boldsymbol{x}}_{0}=\dot{\boldsymbol{y}}_{0}$. The incremental control command is introduced:

$$
\Delta \boldsymbol{u}=\boldsymbol{u}-\boldsymbol{u}_{0}
$$


If the controller update rate is sufficiently high, the approximation $\boldsymbol{x}-\boldsymbol{x}_{0} \approx \mathbf{0}$ is applicable. Hence, Eq. (5) does not depend on $\boldsymbol{A}_{0}$ anymore:

$$
\dot{\boldsymbol{y}} \approx \dot{\boldsymbol{y}}_{0}+\boldsymbol{C}_{0} \boldsymbol{B}_{0} \Delta \boldsymbol{u}
$$

For a commanded derivative of the output, respectively pseudo-control signal, $\dot{y}=\dot{y}_{C}=v$ the required increment of the input signal can be found by rearranging Eq. (7):

$$
\Delta \boldsymbol{u} \approx\left(\boldsymbol{C}_{0} \boldsymbol{B}_{0}\right)^{-1}\left(\boldsymbol{v}-\dot{\boldsymbol{y}}_{0}\right)
$$

or if $\boldsymbol{C}_{0} \boldsymbol{B}_{0}$ is non-square (number of outputs not equal to the number of inputs), a minimum norm solution, minimizing $\Delta \boldsymbol{u}^{T} \boldsymbol{W} \Delta \boldsymbol{u}$, can be found:

$$
\Delta \boldsymbol{u} \approx\left(\boldsymbol{C}_{0} \boldsymbol{B}_{0}\right)^{T}\left(\boldsymbol{C}_{0} \boldsymbol{B}_{0}\left(\boldsymbol{C}_{0} \boldsymbol{B}_{0}\right)^{T}\right)^{-1}\left(\boldsymbol{v}-\dot{\boldsymbol{y}}_{0}\right),
$$

where $\boldsymbol{W}$ is a diagonal weighting matrix.

The pseudo-control signal consists of a reference and a controller contribution:

$$
v_{\dot{y}_{i}}=v_{\dot{y}_{i}, R}+v_{\dot{y}_{i}, P I}
$$

In the next subsection, the pseudo-control signal contribution of the reference model (index $R$ ) and the linear PI controller (index $P I$ ) is presented.

\section{B. Reference Model and Linear Control}

In contrast to the theory of feedback linearization, it is beneficial for flight control applications not to use the integrated variables of the pseudo control signal $v$ for the outer control loops. Instead, other control variables whose physical impact on the aircraft motion is most important are used. This requires a cascaded reference model where each cascade assumes a relative degree of one of the system. In between of each cascade the reference control variable has to be converted into the commanded control variable of the next cascade. Also, a pseudo control hedging for the outer cascades is required [36].

Common control variables for the attitude are the flight path bank angle $\mu_{K}$, the flight path angle of attack $\alpha_{K}$ and the flight path sideslip angle $\beta_{K}$ [22, 25, 37]. Additionally, the tracking variables flight path velocity $V_{K}$, flight path angle $\gamma$ and flight path azimuth $\chi$ are used for the outer control loop. Inside the reference model of each control variable, the reference of the control variable as well as the reference derivative of the control variable can be limited. This so-called dynamic saturation is implemented according to [37].

The contribution of the reference model to the pseudo control signal

$$
v_{\dot{y}_{i, R}}=\frac{1}{T_{y_{i}}}\left(y_{i, C}-y_{i, R}\right)
$$

is calculated with a first order reference model

$$
v_{\dot{y}_{i, R}}=\dot{y}_{i}=\frac{1}{T_{y_{i}}}\left(y_{i, C}-y_{i, R}\right)-v_{\dot{y}_{i, h}},
$$

where $v_{\dot{y}_{i, h}}$ is the pseudo-control hedging signal

$$
v_{\dot{y}_{i}, h}=v_{\dot{y}_{i}}-\dot{y}_{0}
$$

which is used to compensate differences between the reference signal and the measured respectively estimated signal in the reference model (Eq. (12)). Those differences will inevitably occur if there is dynamics between $v_{\dot{y}_{i}}-\dot{y}_{0}$. This is always the case for the inner control loop if the actuator dynamics is not inverted and for the outer control loops if the reference models are multiple first order reference models instead of one higher order reference models are used. In this case, the negligence of the pseudo-control signal could lead to bad performance or even instability.

The portion of the PI controller to the pseudo-control signal $v_{\dot{y}_{i}, P I}$ is needed to make the outputs following the reference if deviations occur:

$$
v_{\dot{y}_{i}, P I}=K_{y, P}\left(y_{R}-y\right)+K_{y, I} \int\left(y_{R}-y\right) \mathrm{d} t
$$




\section{Inner Loop: Angular Velocity}

For the inversion and control of the inner control loop the angular velocity of the body $b$ relative to the flat earth axis system $g$ in body-fixed coordinates $\omega_{b}^{g b}$ is chosen. Since $\omega_{b}^{g b}$ is not only an output but also a state, Eq. (7) is simplified to

$$
\Delta \boldsymbol{u} \approx \boldsymbol{B}_{0}^{-1}\left(v_{\dot{\omega}_{b, 0}^{g b}}-\dot{\boldsymbol{\omega}}_{b}^{g b}\right)=\left(\left.\frac{\partial}{\partial \boldsymbol{u}}(\boldsymbol{g}(\boldsymbol{x}, \boldsymbol{u}))\right|_{\boldsymbol{x}=\boldsymbol{x}_{0}, \boldsymbol{u}=\boldsymbol{u}_{0}}\right)^{-1}\left(\boldsymbol{v}_{\dot{\omega}_{b, 0}^{g b}}-\dot{\boldsymbol{\omega}}_{b}^{g b}\right)
$$

\section{Middle Control Loop: Attitude}

The attitude is controlled with the flight-path bank angle, angle of attack and sideslip angle $\mu_{k}, \alpha_{K}$ and $\beta_{K}$. As the inner control loop is controlled with the angular velocities $\omega_{b, C}^{g b}$, the derivation of the angles $\mu_{K}, \alpha_{K}$ and $\beta_{K}$ has to be converted into the angular velocities $\omega_{b}^{g b}$ :

$$
\omega_{b, C}^{g b}=\left(\begin{array}{c}
p \\
q \\
r
\end{array}\right)=\omega_{b}^{g k}+\omega_{b, C}^{k b}
$$

where

$$
\omega_{b, C}^{k b}=\left(\begin{array}{ccc}
\cos \alpha_{K} \cos \beta_{K} & 0 & \sin \alpha_{K} \\
\sin \beta_{K} & 1 & 0 \\
\sin \alpha_{K} \cos \beta_{K} & 0 & -\cos \alpha_{K}
\end{array}\right)\left(\begin{array}{c}
v_{\mu_{K}} \\
v_{\dot{\alpha}_{K}} \\
v_{\beta_{K}}
\end{array}\right)
$$

and

$$
\boldsymbol{\omega}_{b}^{g k}=\boldsymbol{M}_{b k}\left(\mu_{K}, \alpha_{K}, \beta_{K}\right) \cdot \boldsymbol{\omega}_{k}^{g k}=\boldsymbol{M}_{b k}\left(\mu_{K}, \alpha_{K}, \beta_{K}\right) \cdot\left(\begin{array}{cc}
0 & -\sin \gamma \\
1 & 0 \\
0 & \cos \gamma
\end{array}\right)\left(\begin{array}{l}
\dot{\gamma} \\
\dot{\chi}
\end{array}\right) .
$$

The rotation matrix $\boldsymbol{M}_{b k}\left(\mu_{K}, \alpha_{K}, \beta_{K}\right)$ is defined in the appendix. It requires that $\alpha_{K}, \beta_{K}$ and $\mu_{K}$ can be determined by measurement, estimation or observation. If there is no wind, the aircraft velocity relative to earth and relative to the air is equal so that $\alpha_{K}=\alpha$ and $\beta_{K}=\beta$. While the aerodynamic angles $\alpha$ and $\beta$ are very common measured quantities the determination of the flight-path bank angle $\mu_{K}$ is more complicated:

$$
\mu_{K}=\arcsin \left(\frac{\sin \gamma \sin \beta_{K}+\sin \Phi \cos \Theta}{\cos \beta_{K} \cos \gamma}\right) .
$$

Equation 19 is obtained by the comparison of the element $(2,3)$ of the matrices

$$
\boldsymbol{M}_{b g}(\Phi, \Theta, \Psi)=\boldsymbol{M}_{b k}\left(\mu_{K}, \alpha_{K}, \beta_{K}\right) \boldsymbol{M}_{k g}(\gamma, \chi) .
$$

The rotation matrices $\boldsymbol{M}_{b g}(\Phi, \Theta, \Psi)$ and $\boldsymbol{M}_{k g}(\gamma, \chi)$ are defined in the appendix.

\section{Outer Control Loop: Flight Path}

For the flight path control the tracking outputs flight path velocity $V_{K}$, flight-path azimuth $\chi$ and flight path angle $\gamma$ are used. Again, the pseudo-control signal of the flight path control $\left(v_{\dot{V}_{K}} v_{\dot{\gamma}} v_{\dot{\chi}}\right)_{C}^{T}$ has to be transformed into the commanded attitude $\left(\mu_{K} \alpha_{K} \beta_{K}\right)_{C}^{T}$.

The derivation of the flight path (index fp) outputs $\dot{V}_{K}, \dot{\chi}, \dot{\gamma}$ is a nonlinear function of the state vector depending on external forces caused by the aerodynamics, propulsion and gravity: 


$$
\left(\begin{array}{c}
\dot{V}_{K} \\
\dot{\chi} \\
\dot{\gamma}
\end{array}\right)=\frac{\partial \boldsymbol{h}_{\mathrm{fp}}}{\partial \boldsymbol{x}}(\boldsymbol{f}(\boldsymbol{x})+\boldsymbol{g}(\boldsymbol{x}, \boldsymbol{u})
$$

As the functions are difficult to invert analytically, an incremental inversion with the linear approximation of the function is implemented:

$$
\left(\begin{array}{c}
\Delta \dot{V}_{K} \\
\Delta \dot{\chi} \\
\Delta \dot{\gamma}
\end{array}\right) \approx \frac{\partial}{\partial \boldsymbol{y}_{\mathrm{att}}}\left(\left.\frac{\partial \boldsymbol{h}_{\mathrm{fp}}}{\partial \boldsymbol{x}}(\boldsymbol{f}(\boldsymbol{x})+\boldsymbol{g}(\boldsymbol{x}, \boldsymbol{u}))\right|_{\boldsymbol{x}_{0}, \boldsymbol{u}_{0}} \Delta \boldsymbol{y}_{\mathrm{att}} \quad \text { with } \quad \boldsymbol{y}_{\mathrm{att}}=\left(\begin{array}{c}
\mu_{K} \\
\alpha_{K} \\
\beta_{K}
\end{array}\right) .\right.
$$

The obtained matrix can be inverted numerically in order to transform the pseudo-control signal of the flight path control to the commanded attitude:

$$
\left(\begin{array}{c}
\mu_{K} \\
\alpha_{K} \\
\beta_{K}
\end{array}\right)_{C}=\left(\begin{array}{c}
\mu_{K} \\
\alpha_{K} \\
\beta_{K}
\end{array}\right)_{0}+\left(\begin{array}{c}
\Delta \mu_{K} \\
\Delta \alpha_{K} \\
\Delta \beta_{K}
\end{array}\right)_{C} \approx\left(\begin{array}{c}
\mu_{K} \\
\alpha_{K} \\
\beta_{K}
\end{array}\right)_{0}+\left(\frac{\partial}{\partial \boldsymbol{y}_{\mathrm{att}}}\left(\left.\frac{\partial \boldsymbol{h}_{\mathrm{fp}}}{\partial \boldsymbol{x}}(\boldsymbol{f}(\boldsymbol{x})+\boldsymbol{g}(\boldsymbol{x}, \boldsymbol{u}))\right|_{\boldsymbol{x}_{0}, \boldsymbol{u}_{0}}\right)^{-1}\left(\begin{array}{c}
v_{\dot{V}_{K}}-\dot{V}_{K} \\
v_{\dot{\chi}}-\dot{\chi} \\
v_{\dot{\gamma}}-\dot{\gamma}
\end{array}\right) .\right.
$$

This method assumes that the measurement, estimation or observation of the derivation of the flight path velocity $\dot{V}_{K}$, the flight path angle $\dot{\gamma}$ and the flight-path azimuth $\dot{\chi}$ are available. The derivation of the flight path angle and the flight-path azimuth can be calculated with respect to the derivation of the flight path velocity which commonly is available:

$$
\begin{gathered}
\gamma=-\arcsin \frac{w_{K g}}{\left|V_{K}\right|}=\arctan \left(\frac{-w_{K g}}{\sqrt{\left(u_{K g}^{2}+v_{K g}^{2}\right.}}\right), \quad \dot{\gamma}=\frac{-\dot{w}_{K g}\left(u_{K g}^{2}+v_{K g}^{2}\right)+w_{K g}\left(u_{K g} \dot{u}_{K g}+v_{K g} \dot{v}_{K g}\right)}{\left(u_{K g}^{2}+v_{K g}^{2}+w_{K g}^{2}\right) \sqrt{u_{K g}^{2}+v_{K g}^{2}}}, \\
\chi=\arctan 2\left(u_{K g}, v_{K g}\right), \quad \dot{\chi}=\frac{\dot{v}_{K g} v_{K g}-v_{K g} \dot{v}_{K g}}{v_{K g}^{2}+u_{K g}^{2}} .
\end{gathered}
$$

\section{Overall Control Strategy}

The overall control strategy resulting of the combination of the explained parts is shown in Fig. 7. The controller update rate is $100 \mathrm{~Hz}$ whereas the update rate for the linearization of $\boldsymbol{B}_{0}$ is $25 \mathrm{~Hz}$.

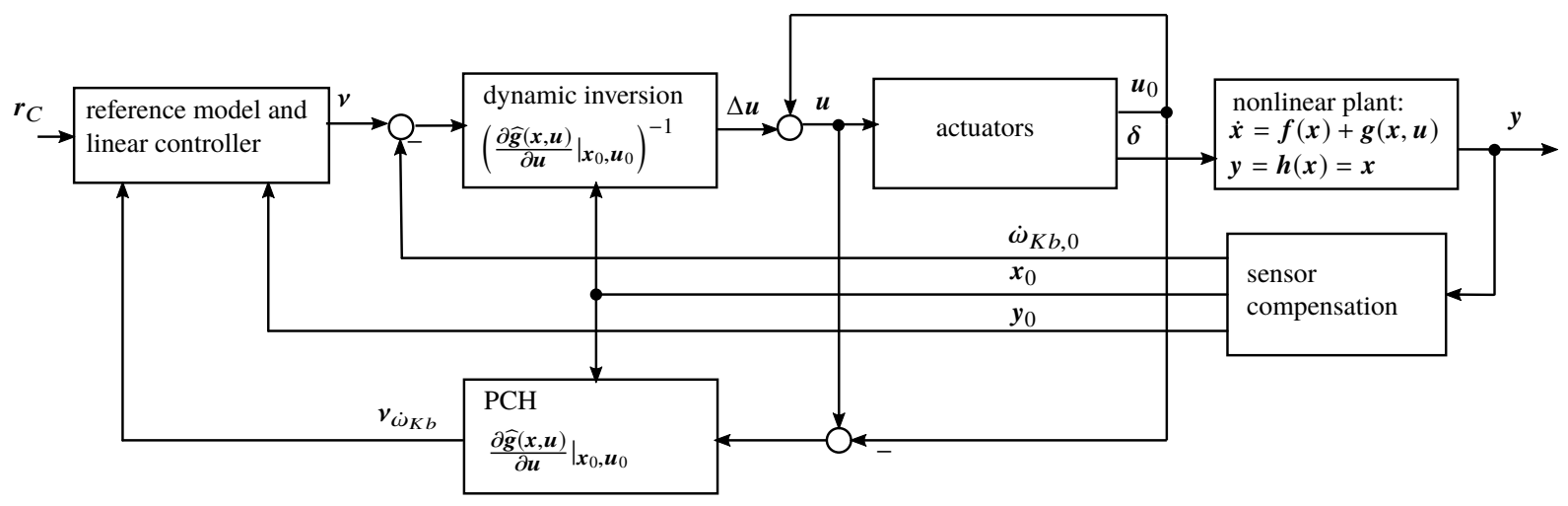

Fig. 7 Illustration of the adaptive control strategy with incremental nonlinear dynamic inversion and neural network. 


\section{Simulation Results}

In this section, a partial failure scenario of the aircraft in landing configuration is defined. Then, the simulated aircraft reactions with INDI on this failure scenario are presented.

\section{A. Failure Scenario}

As explained in section [III] a failure of a micro-compressor of the high-lift system is critical. It is assumed to be most critical for the lowest allowed airspeed which is $V=44.25 \mathrm{~m} / \mathrm{s}$. Each compressor on one wing side has a different impact on the aerodynamic coefficients (see Fig. 6). Thus, it is not clear which single compressor failure is the most critical. Moreover, only two of six compressor failures are based on CFD data whereas the others are modeled and underlie additional model uncertainties. For the fourth compressor failure CFD data are given. Additionally, it significantly decreases both the lift and rolling coefficient which is assumed to be an especially critical case. Consequently, the regarded failure scenario is an instant drop of the local $C_{\mu}$ of the fourth micro-compressor to zero during approach at $V=44.25 \mathrm{~m} / \mathrm{s}$ and constant altitude without wind.

The trim conditions for steady horizontal flight with no sideslip angle are defined by:

$$
\dot{\boldsymbol{V}}_{b}^{g b}=\dot{\boldsymbol{V}}_{K b}=\mathbf{0}, \quad V=44.25 \mathrm{~m} / \mathrm{s}, \quad \omega_{b}^{g b}=\mathbf{0}, \quad \Phi=0^{\circ}, \quad \Theta=0^{\circ}, \quad \alpha=\alpha_{\text {Trim }}, \quad \beta=0^{\circ}, \quad \gamma=0^{\circ} .
$$

Moreover, the control surface deflections are supposed to be zero, the jet momentum coefficient is fixed and the thrust setting is unknown:

$$
\delta_{\xi}=\delta_{\eta}=\delta_{\zeta}=0, \quad C_{\mu}=0.0306, \quad \delta_{t}=\delta_{t, \text { Trim }} .
$$

The command $\boldsymbol{r}_{C}$ during the scenario is $\gamma_{C}=0$ and $\chi_{C}=0$.

From these conditions, the aircraft model is trimmed by an iterative process so that all unknown states, outputs and inputs are found. These are for instance:

$$
\alpha=2.4^{\circ}, \quad \delta_{t}=0.30
$$

\section{B. Results of the Adaptive Controller}

The aircraft reaction to the compressor failure (injected at $t=2 \mathrm{~s}$ ) is shown in Fig. 8 to Fig. 11. In the figures, the reference signal of each state or output is also shown. Obviously, the actual states or outputs are almost identical to the reference signal. However, the reference signal $y_{R}$ is not the commanded signal $y_{C}$ signal (see Eq. (11) and Eq. (12)). This is also due to the pseudo-control hedging which was presented in section IV Significant deviations between the reference signal and the actual control variable only occur if the measured feedback of the control variables and the derivative of the angular velocity vector is error-prone. That is the case if the block sensor compensation in Fig. 7 is not equal to 1. In reality, this is the case as sensors suffer from noise, bias and other errors. For good control performance, these errors are supposed to be minimized. The occurring deviation between reference signal and actual control variable is corrected by the PI controller. However, the reference will still not be perfectly tracked in that case.

Figure 8 shows the control surface reactions on the compressor failure after two seconds. As the delay of the feedback system is $0.09 \mathrm{~s}$, the actuator states start to change after $2.09 \mathrm{~s}$. Both the aileron and the elevator deflections immediately change with the maximum rate to compensate the roll moment and the loss of lift. The aileron reaches the maximum deflection of $34^{\circ}$ one second after the compressor failure and finally converge to a deflection of $18^{\circ}$. The maximum elevator deflection of $-5^{\circ}$ is clearly smaller than the maximum aileron deflection. The maximum would be somewhat higher if there were no dynamic saturation of the rate of the angle of attack in the reference model. The steady elevator deflection is only about $2^{\circ}$. The maximum rudder deflection to compensate the yawing moment is also about $4^{\circ}$, however, it does not change with maximum rate. After a few seconds the rudder deflection remains constant at slightly less than $1^{\circ}$.

The angular velocity immediately changes after two seconds when the failure occurs which is shown in Fig. 9 . Obviously, the rolling moment is significantly higher than the yawing moment. The initial pitching moment is zero. About $7 \mathrm{~s}$ after the failure the angular velocities are almost steady and tend to zero again. After a delay of approximately $0.1 \mathrm{~s}$ after the failure, a positive pitch rate occurs which is because of the negative elevator deflection resulting in an increased angle of attack (see Fig. 10. 
According to figure 10 the angle of attack changes from $2.4^{\circ}$ to $3.4^{\circ}$ which compensates the loss of lift caused by the failure of the compressor. $2.5 \mathrm{~s}$ after the failure, the angle of attack reaches its maximum of $4.2^{\circ}$. The maximum flight path bank angle of $2.1^{\circ}$ is reached $0.9 \mathrm{~s}$ after the failure. The aircraft then remains slightly banked to the left in order to finish correcting the flight path azimuth. The sideslip angle is always smaller than $0.2^{\circ}$.

Figure 11 shows that the flight path angle decreases directly as the loss of lift causes a vertical acceleration (see Eq. (24)). The reaction of the flight path azimuth starts delayed. The magnitude of both control variables is one about $0.5^{\circ}$. However, since the time constant of the flight path angle reference model is relatively large, it takes about $10 \mathrm{~s}$ until the control variables tend to zero again.

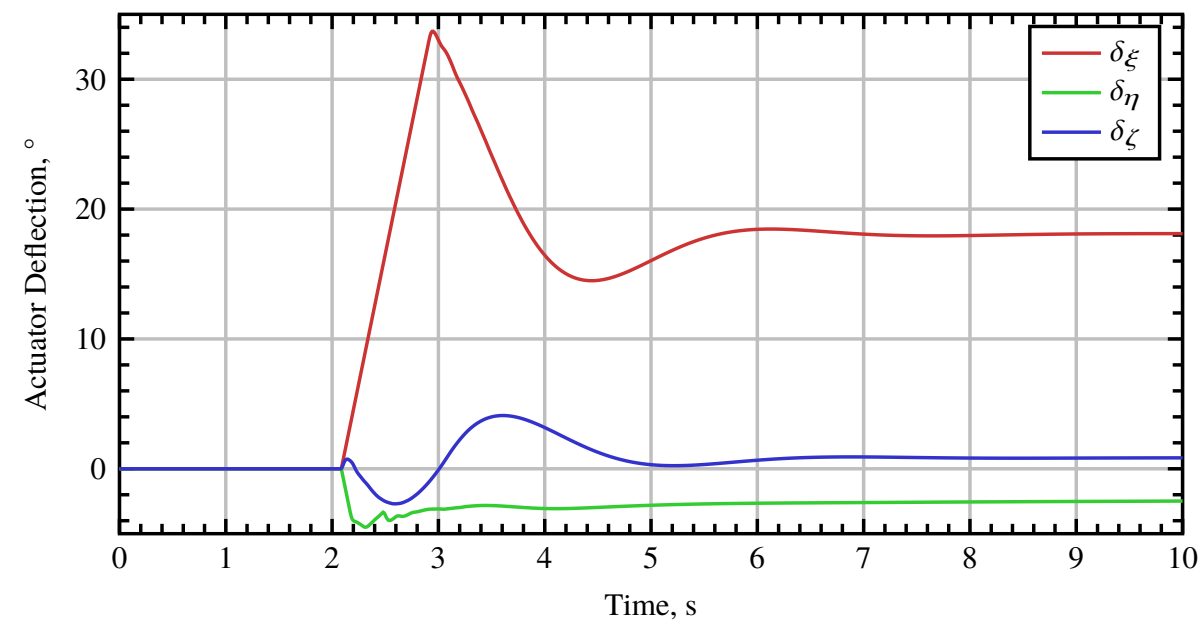

Fig. 8 Control surface deflection due to a compressor failure of the active high-lift system after 2 seconds.

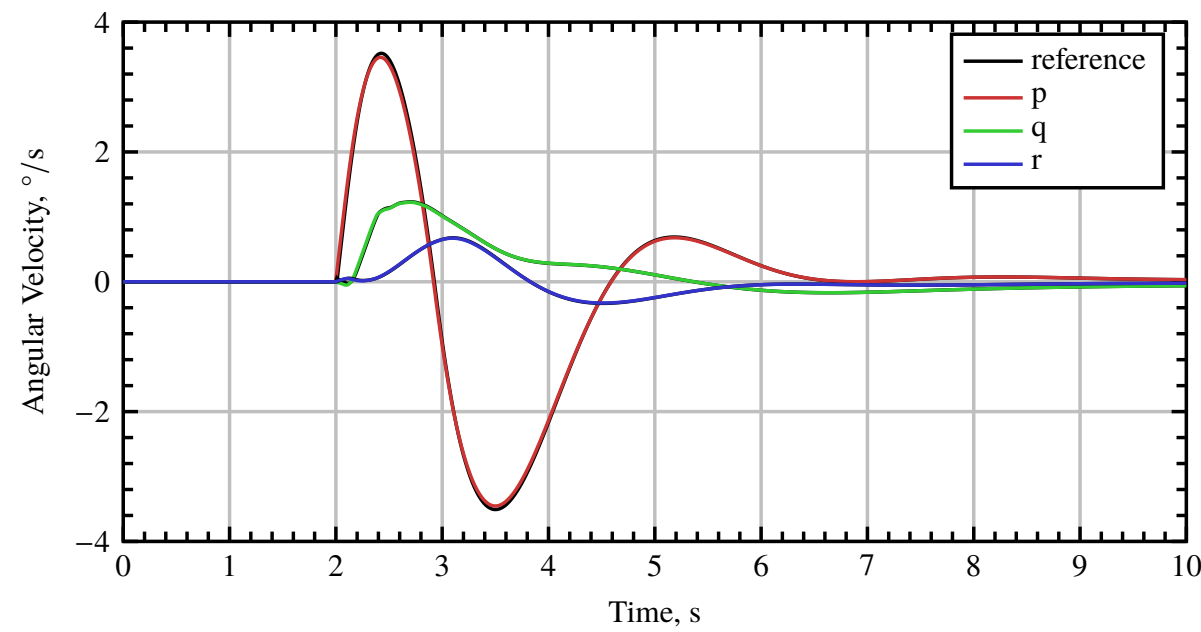

Fig. 9 Angular velocity due to a compressor failure of the active high-lift system after 2 seconds. 


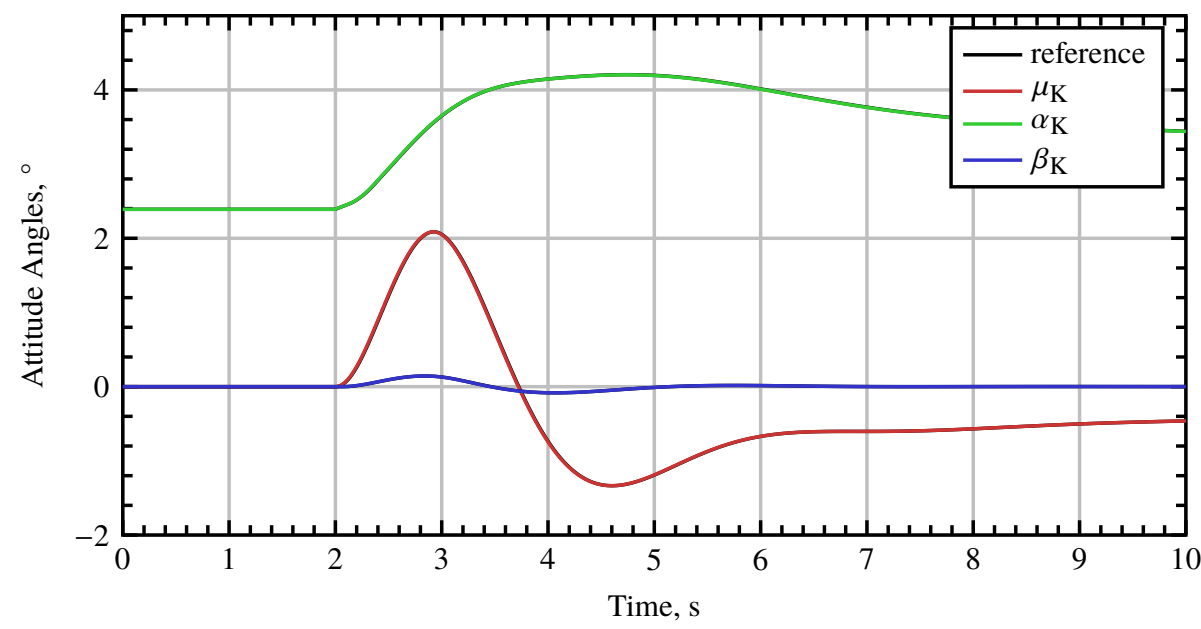

Fig. 10 Attitude angles due to a compressor failure of the active high-lift system after 2 seconds.

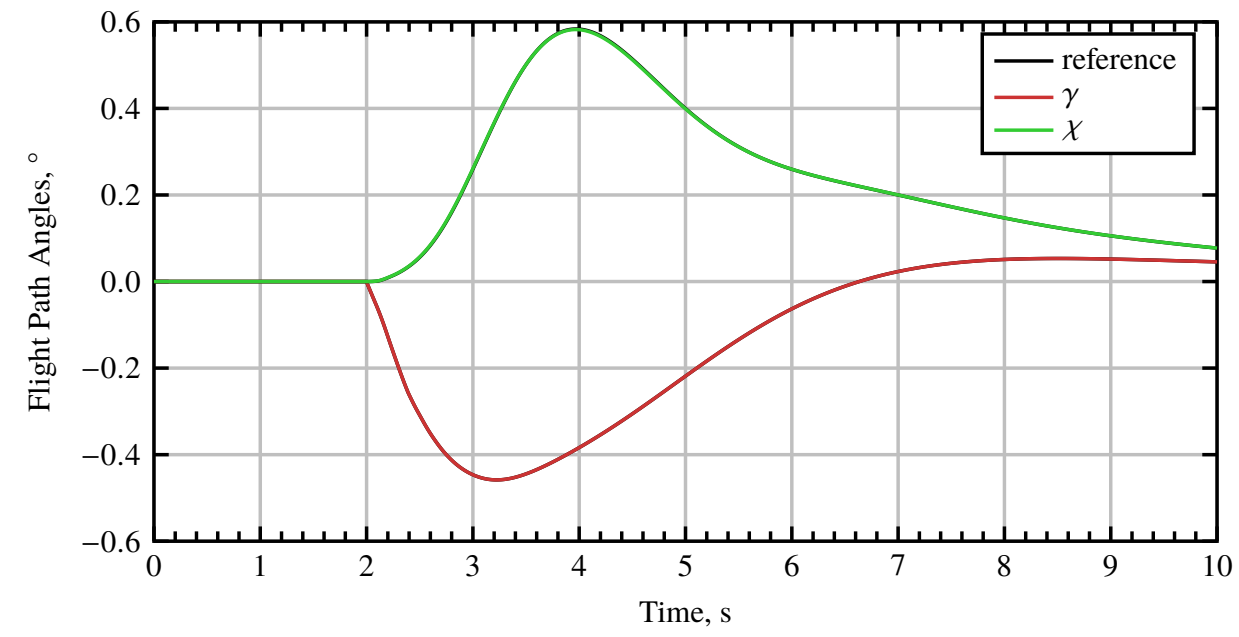

Fig. 11 Flight path angles due to a compressor failure of the active high-lift system after 2 seconds.

The presented simulations results assume perfect sensors. If noise and bias are added to the measured signals, the magnitude of the signals, the number as well as the number of oscillations increase. However, in order to properly analyze the influence of sensor errors, both high fidelity sensor models and appropriate sensor data fusion would be required. Both are not yet implemented and should be addressed in future works.

\section{Conclusion}

This work presented an INDI controller for adaptive nonlinear control with application for aircraft with a partly failing active high-lift system. A non-compensated failure of one compressor of the active high-lift system would cause critical reactions of the aircraft during approach because of a loss of lift and a resulting moment. In this scenario the IDNI controller behaves very well and automatically stabilizes the aircraft within a few seconds using the conventional control surfaces. During the transient response the states of the rigid body only slightly change.

However, to control such a scenario, a relatively large and quick (rate limit reached) aileron deflection is required. The aileron deflection almost reaches its limit position. This is why this scenario could still be critical when additional aileron deflection for maneuvers or atmospheric disturbance are required. Moreover, a second nearby compressor failure will inevitably lead to a fatal failure of the system since the maximum aileron deflection will be reached. Another problem could be the increased angle of attack to compensate the loss of lift because the safety against stall is decreased. 
As a consequence, it could be beneficial to use all compressors as control inputs. Thus, the complete potential for control effectiveness of the aircraft could be used. With this, the steady aileron deflection during such a scenario could be reduced and the angle of attack remains constant. A direct lift control with the active high-lift system would also increase the maneuverability. Possibly multiple compressor failures could be compensated too.

Furthermore, the stability of the aircraft with IDNI controller should be investigated. That also requires investigations on signal noise in the feedback loop.

To provide adaptivity of the controller against changes in the control effectiveness, an adaption law for the input matrix could be used.

Rotation matrices:

\section{Appendix}

$$
\begin{aligned}
\boldsymbol{M}_{b k}\left(\mu_{K}, \alpha_{K}, \beta_{K}\right) & =\left(\begin{array}{ccc}
\cos \alpha_{K} & 0 & -\sin \alpha_{K} \\
0 & 1 & 0 \\
\sin \alpha_{K} & 0 & \cos \alpha_{K}
\end{array}\right)\left(\begin{array}{ccc}
\cos \beta_{K} & -\sin \beta_{K} & 0 \\
\sin \beta_{K} & \cos \beta_{K} & 0 \\
0 & 0 & 1
\end{array}\right)\left(\begin{array}{ccc}
1 & 0 & 0 \\
0 & \cos \mu_{K} & \sin \mu_{K} \\
0 & -\sin \mu_{K} & \cos \mu_{K}
\end{array}\right) \\
& =\left(\begin{array}{ccc}
\cos \alpha_{K} \cos \beta_{K} & -\cos \alpha_{K} \sin \beta_{K} \cos \mu_{K}+\sin \alpha_{K} \sin \mu_{K} & -\cos \alpha_{K} \sin \beta_{K} \sin \mu_{K}-\sin \alpha_{K} \cos \mu_{K} \\
\sin \beta_{K} & \cos \beta_{K} \cos \mu_{K} & \cos \beta_{K} \sin \mu_{K} \\
\sin \alpha_{K} \cos \beta_{K} & -\sin \alpha_{K} \sin \beta_{K} \cos \mu_{K}-\cos \alpha_{K} \sin \mu_{K} & -\sin \alpha_{K} \sin \beta_{K} \sin \mu_{K}+\cos \alpha_{K} \cos \mu_{K}
\end{array}\right)
\end{aligned}
$$

$$
\begin{aligned}
\boldsymbol{M}_{b g}(\Phi, \Theta, \Psi) & =\left(\begin{array}{ccc}
1 & 0 & 0 \\
0 & \cos \Phi & \sin \Phi \\
0 & -\sin \Phi & \cos \Phi
\end{array}\right)\left(\begin{array}{ccc}
\cos \Theta & 0 & -\sin \Theta \\
0 & 1 & 0 \\
\sin \Theta & 0 & \cos \Theta
\end{array}\right)\left(\begin{array}{ccc}
\cos \Psi & \sin \Psi & 0 \\
-\sin \Psi & \cos \Psi & 0 \\
0 & 0 & 1
\end{array}\right) \\
& =\left(\begin{array}{ccc}
\cos \Theta \cos \Psi & \cos \Theta \sin \Psi & \sin \Theta \\
-\cos \Phi \sin \Psi+\sin \Phi \sin \Theta \cos \Psi & \cos \Phi \cos \Psi+\sin \Phi \sin \Theta \sin \Psi & \sin \Phi \cos \Theta \\
\sin \Phi \sin \Psi+\cos \Phi \sin \Theta \cos \Psi & -\sin \Phi \cos \Psi+\cos \Phi \sin \Theta \sin \Psi & \cos \Phi \cos \Theta
\end{array}\right)
\end{aligned}
$$

$$
\begin{aligned}
\boldsymbol{M}_{k g}(\gamma, \chi) & =\left(\begin{array}{ccc}
\cos \gamma & 0 & -\sin \gamma \\
0 & 1 & 0 \\
\sin \gamma & 0 & \cos \gamma
\end{array}\right)\left(\begin{array}{ccc}
\cos \chi & \sin \chi & 0 \\
-\sin \chi & \cos \chi & 0 \\
0 & 0 & 1
\end{array}\right) \\
& =\left(\begin{array}{ccc}
\cos \gamma \cos \chi & \cos \gamma \sin \chi & -\sin \gamma \\
-\sin \chi & \cos \chi & 0 \\
\sin \gamma \cos \chi & \sin \gamma \sin \chi & \cos \gamma
\end{array}\right)
\end{aligned}
$$

\section{Acknowledgments}

This work has been supported by the provision of particular CFD results for the spanwise aerodynamics of the wing with DLR's TAU code by Dennis Keller. The reference aircraft design has been developed by Wolfgang Heinze with PrADO. The research is conducted within the framework of the Sonderforschungsbereich 880.

\section{References}

[1] Radespiel, R., and Heinze, W., "SFB 880: fundamentals of high lift for future commercial aircraft," CEAS Aeronautical Journal, Vol. 5, No. 3, 2014, pp. 239-251. 
[2] Werner-Spatz, C., Heinze, W., Horst, P., and Radespiel, R., "Multidisciplinary conceptual design for aircraft with circulation control high-lift systems," CEAS Aeronautical Journal, Vol. 3, No. 2-4, 2012, pp. 145-164.

[3] Seume, J. R., Teichel, S., Burnazzi, M., Schwerter, M., Behr, C., Rudenko, A., Schmitz, A., Dorbaum, M., and Atalayer, C., "SFB 880 - efficient high lift," Proceedings of the 62nd Deutscher Luft- und Raumfahrtkongress 2013, Stuttgart, 2013.

[4] Diekmann, J. H., "Flight Mechanical Challenges of STOL Aircraft Using Active High-Lift," Proceedings of 35th AIAA Applied Aerodynamics Conference, American Institute of Aeronautics and Astronautics, 2017.

[5] Keller, D., and Rudnik, R., "Numerical investigations of aerodynamic properties of a propeller blown circulation control system on a high wing aircraft," CEAS Aeronautical Journal, Vol. 7, No. 3, 2016, pp. 441-454.

[6] Keller, D., and Rudnik, R., "Numerical investigation of engine effects on a transport aircraft with circulation control," Journal of Aircraft, 2015.

[7] Burnazzi, M., and Radespiel, R., "Design and analysis of a droop nose for coanda flap applications," Journal of Aircraft, Vol. 51, No. 5, 2014, pp. 1567-1579.

[8] Radespiel, R., Burnazzi, M., Casper, M., and Scholz, P., "Active flow control for high lift with steady blowing," The Aeronautical Journal, Vol. 120, No. 1223, 2016, pp. 171-200.

[9] Henri, C., "Device for deflecting a stream of elastic fluid projected into an elastic fluid," , Sep. 1 1936. US Patent 2,052,869.

[10] Calise, A. J., and Rysdyk, R. T., "Nonlinear adaptive flight control using neural networks," IEEE Control Systems, Vol. 18, No. 6, 1998, pp. 14-25.

[11] Rysdyk, R., and Calise, A. J., "Robust Nonlinear Adaptive Flight Control for Consistent Handling Qualities," IEEE Transactions on Control Systems Technology, Vol. 13, No. 6, 2005, pp. 896-910.

[12] Nguyen, N. T., and Jacklin, S. A., Stability, Convergence, and Verification and Validation Challenges of Neural Net Adaptive Flight Control, Springer Berlin Heidelberg, Berlin, Heidelberg, 2010, pp. 77-110.

[13] Krüger, T., Schnetter, P., Placzek, R., and Vörsmann, P., "Nonlinear adaptive flight control using sliding mode online learning," The 2011 International Joint Conference on Neural Networks, 2011, pp. 2897-2904.

[14] Schnetter, P., and Krüger, T., "Compensation of significant parametric uncertainties using sliding mode online learning," 2013 IEEE Aerospace Conference, 2013, pp. 1-9.

[15] Johnson, E., Calise, A., El-Shirbiny, H., and Eysdyk, R., "Feedback linearization with Neural Network augmentation applied to X-33 attitude control," AIAA Guidance, Navigation, and Control Conference and Exhibit, American Institute of Aeronautics and Astronautics (AIAA), 2000.

[16] Hovakimyan, N., Kim, N., Calise, A. J., Prasad, J. V. R., and Corban, E., “Adaptive Output Feedback for High-Bandwidth Control of an Unmanned Helicopter," Proceedings of the AIAA Guidance, Navigation, and Control Conference, 2001.

[17] Kim, N., "Improved Methods in Neural Network-Based Adaptive Output Feedback Control with Applications to Flight Control," $\mathrm{Ph}$.D. thesis, School of Aerospace Engineering, Georgia Institute of Technology, 2003.

[18] Lee, S., Ha, C., and Kim, B. S., "Adaptive nonlinear control system design for helicopter robust command augmentation," Aerospace Science and Technology, Vol. 9, No. 3, 2005, pp. 241 - 251.

[19] Pullum, L. L., Taylor, B. J., and Darrah, M. A., Guidance for the Verification and Validation of Neural Networks, Emerging Technologies, Wiley, 2007.

[20] Schumann, J., and Liu, Y. (eds.), Applications of Neural Networks in High Assurance Systems, Springer Berlin Heidelberg, 2010.

[21] Johnson, E. N., "Limited Authority Adaptive Flight Control,” Ph.D. thesis, Georgia Institute of Technology, 2000.

[22] Chen, H. B., and Zhang, S. G., "Robust dynamic inversion flight control law design," 2008 2nd International Symposium on Systems and Control in Aerospace and Astronautics, 2008, pp. 1-6.

[23] Sieberling, S., Chu, Q. P., and Mulder, J. A., "Robust Flight Control Using Incremental Nonlinear Dynamic Inversion and Angular Acceleration Prediction," Journal of Guidance, Control, and Dynamics, Vol. 33, No. 6, 2010, pp. $1732-1742$.

[24] Acquatella, P. B., Falkena, W., van Kampen, E.-J., and Chu, Q. P., "Robust nonlinear spacecraft attitude control using incremental nonlinear dynamic inversion," AIAA Guidance, Navigation, and Control Conference, 2012, pp. 1-20. 
[25] Acquatella, P. B., van Ekeren, W., and Chu, Q. P., "PI(D) tuning for Flight Control Systems via Incremental Nonlinear Dynamic Inversion," CoRR, Vol. abs/1701.08981, 2017.

[26] Gerhold, T., "Overview of the hybrid RANS code TAU," MEGAFLOW-Numerical Flow Simulation for Aircraft Design, Springer, 2005, pp. 81-92.

[27] Diekmann, J. H., "Flugmechanische Charakteristika von propellergetriebenen Flugzeugen mit aktiven Hochauftriebssystemen," Ph.D. thesis, TU Braunschweig, 2017.

[28] Horst, P., Sommerwerk, K., Neuert, N., Krukow, I., Keller, D., Diekmann, J. H., and Rang, J., "Flight Dynamics Investigation of an Active High-Lift Aircraft," Prodeeding of the 35th AIAA Applied Aerodynamics Conference, American Institute of Aeronautics and Astronautics, 2017.

[29] Diekmann, J. H., "Analysis of trimmable conditions for a civil aircraft with active high-lift system," CEAS Aeronautical Journal, Vol. 6, No. 1, 2015, pp. 109-120.

[30] Quigley, H. C., and Innis, R. C., "Handling qualities and operational problems of a large four-propeller STOL transport airplane," , 1963.

[31] Keller, D., and Rudnik, R., "Integration Aspects of Lift Augmentation Systems on the Aerodynamics of a High-Lift Configuration," SFB 880 - Fundamentals of high-lift for future commertial aircraft, Biennial Report, edited by R. Radespiel and R. Semaan, TU Braunschweig - Campus Forschungsflughafen, 2015, pp. 145-156.

[32] Diekmann, J. H., Keller, D., Faez, E., Rudnik, R., and Gollnick, V., "Flight mechanics model for spanwise lift and rolling moment distributions of a segmented active high-lift wing,", 2017.

[33] Slotine, J.-J., and Li, W., Applied Nonlinear Control, PRENTICE HALL, 1990.

[34] Khalil, H. K., Nonlinear systems, third edition ed., Pearson Education, Prentice Hall, Upper Saddle River, NJ, 2002.

[35] Bacon, J. B., and Ostroff, J. A., "Reconfigurable flight control using nonlinear dynamic inversion with a special accelerometer implementation," AIAA Guidance, Navigation, and Control Conference and Exhibit, 2000, p. 4565.

[36] Johnson, E. N., and Calise, A. J., "Pseudo-control hedging: A new method for adaptive control," Advances in navigation guidance and control technology workshop, Alabama, USA Alabama, USA, 2000, pp. 1-2.

[37] Holzapfel, F., "Nichtlineare adaptive Regelung eines unbemannten Fluggerätes,” Ph.D. thesis, Technische Universität München, Universitätsbibliothek, 2004. 